\title{
Segmentation of Remotely Sensed Images Using Wavelet Features and Their Evaluation in Soft Computing Framework
}

\author{
Mausumi Acharyya, Rajat K. De, Member, IEEE, and Malay K. Kundu, Senior Member, IEEE
}

\begin{abstract}
The present paper describes a feature extraction method based on $M$-band wavelet packet frames for segmenting remotely sensed images. These wavelet features are then evaluated and selected using an efficient neurofuzzy algorithm. Both the feature extraction and neurofuzzy feature evaluation methods are unsupervised, and they do not require the knowledge of the number and distribution of classes corresponding to various land covers in remotely sensed images. The effectiveness of the methodology is demonstrated on two four-band Indian Remote Sensing 1A satellite (IRS-1A) images containing five to six overlapping classes and a three-band SPOT image containing seven overlapping classes.
\end{abstract}

Index Terms-Adaptive basis selection, fuzzy feature evaluation index, $M$-band wavelet packet frames, neural networks, remotely sensed image.

\section{INTRODUCTION}

$\mathbf{T}$ HE SEGMENTATION of different land cover regions in a remotely sensed image is recognized as a complex problem. These images usually have inferior illumination quality and are mainly due to different type of environmental distributions. The natural scene in general contains many objects (regions), e.g., vegetation, water bodies, habitation, concrete structures, open spaces etc., but these regions are mostly not very well separated because of low spatial resolution. Moreover, generally the gray value assigned to a pixel is due to the average reflectance of different types of land covers present in an area that corresponds to a pixel. Assigning unique class levels with certainty for all the pixel is, thus, a genuine problem of remotely sensed images. Fuzzy set theory can provide a way of handling such uncertainties in a better way.

Remotely sensed images may contain information over a large range of scales, the spatial frequency structure and also changes over different regions (i.e., nonperiodic signal). In remote sensing image analysis, it is important to understand how information changes over different scales of imagery. This justifies the use of multiresolution type analysis for such purpose and is most effective using wavelets. Moreover, wavelet theory is well suited in this area of study where signals are complex

Manuscript received December 27, 2001; revised January 28, 2003

M. Acharyya is with the E-Radar Division, Electronics and Radar Development Establishment, Defence Research Development Organization, DRDO Complex, Bangalore 560 093, India (e-mail: mau_ach@yahoo.co.in).

R. K. De and M. K. Kundu are with the Machine Intelligence Unit, Indian Statistical Institute, Kolkata 700 108, India (e-mail: rajat@isical.ac.in; malay@isical.ac.in).

Digital Object Identifier 10.1109/TGRS.2003.815398 and nonperiodic. Furthermore, wavelets are particularly good in describing a scene in terms of the scale of the textures in it.

Texture is an important property of reflective surface. In a remotely sensed image, texture is considered to be the visual impression of coarseness or smoothness caused by the variability or uniformity of image tone. These textural properties of a remotely sensed image are likely to provide valuable information for analysis, where different object regions are treated as different texture classes, i.e., a multitexture segmentation problem.

The wide variety of texture analysis methods that have been developed over the past two decades are reviewed in [1]. Haralick et al. [2] have used gray-level cooccurrence features to analyze remotely sensed images. Rignot and Kwok [3] have analyzed synthetic aperture radar (SAR) images using texture features computed from gray-level cooccurrence matrices. The use of various texture features have been studied for analyzing SAR images by Du [4]. He used the Gabor filters for extracting texture features and successfully segmented SAR images.

Mecocci et al. [5] presented a wavelet-based algorithm combined with a fuzzy c-means classifier. Lindsay et al. [6] used the one-dimensional discrete wavelet transform (DWT) based on Daubechies wavelet filter. A wavelet-based texture feature set is derived in [7]. It consists of the energy of subimages obtained by the overcomplete wavelet decomposition of local areas in SAR images.

Simard et al. [8] studied the use of a decision tree classifier and multiscale texture measures to extract thematic information on the tropical vegetation cover from the Global Rain Forest Mapping (GRFM) mission. The aim of the work in [9] is to show how coastlines can be derived from SAR images by using wavelet and active contour methods. Several other wavelet-based segmentation for geoscience and remote sensing applications have also been reported in the literature [10].

All of these above methods use supervised classification where a priori knowledge about the images is essential. We apply a methodology to carry out this segmentation task where no a priori knowledge about the image is available.

Applications of an octave-band wavelet decomposition scheme for texture segmentation to remotely sensed images have been studied in [5] and [6]-[9]. The octave-band wavelet decomposition [11] provides a logarithmic frequency resolution and is not suitable for the analysis of high-frequency signals with relatively narrow bandwidth. Therefore, the main motivation of the present work is to utilize the decomposition scheme based on $M$-band $(M>2)$ wavelets, which, unlike the standard wavelet, provide a mixture of logarithmic and linear 
frequency resolution [12], [13] and hence able to characterize a texture more efficiently. We conjecture that $M$-band wavelet decomposition may provide better segmentation results than the methods mentioned earlier. But the use of $M$-band wavelet decomposition gives rise to a large number of features, which may incur redundancy and confusion. Therefore, selection of the appropriate features using some feature selection algorithms is essential.

The proposed methodology for segmenting a remotely sensed satellite image has two parts. The first one deals with extraction of texture features using $M$-band wavelet packet frame, followed by their neurofuzzy evaluation for selecting an optimal set of features. Note that the $M$-band $(M>2)$ wavelet transform is a tool for viewing signals at different scales and decomposes a signal by projecting it onto a family of functions generated from a single wavelet basis via its dilations and translations [13]. Neurofuzzy computing [14], which integrates the merits of fuzzy set theory and artificial neural networks (ANNs), enables the feature selection process artificially more intelligent. Incorporation of fuzzy set theory helps one to deal with uncertainties in remotely sensed images in an efficient manner. ANNs are used here for the task of optimization in an adaptive manner.

The paper is organized as follows. Section II presents the mathematical framework of $M$-band wavelets. Section III discusses the filtering technique used in our investigation and the extraction of features. Section IV provides the neurofuzzy feature selection algorithm. Section V analyzes experimental results, and the paper concludes with Section VI.

\section{II. $M$-BAND WAVELET TRANSFORM}

\section{A. M-Band Wavelets}

$M$-band orthonormal wavelets is a direct generalization of the two-band orthogonal wavelets. These $M$-band wavelets are able to zoom in onto narrowband high-frequency components of a signal and have been found to provide better energy compaction than two-band wavelets [15].

An $M$-band wavelet system consists of a scaling function $\phi(x)$ and $(M-1)$ wavelet functions $\psi_{l}(x)$. In discrete form, these functions can be indexed by scale parameter $j$ and translation parameter $k$ and is written as [13]

$$
\begin{aligned}
\phi_{j, k}(x) & =\sum_{k} M^{j / 2} \phi\left(M^{j} x-k\right) \\
\psi_{j, k, l}(x) & =\sum_{k} M^{j / 2} \psi_{l}\left(M^{j} x-k\right), \quad l=1, \ldots, M-1 .
\end{aligned}
$$

The subspaces spanned by the functions $\phi_{j, k}(x)$ and $\psi_{j, k, l}(x)$ be, respectively, defined as $\mathcal{V}_{j}=\overline{\operatorname{span}_{k} \phi_{j, k}} ; \forall k \in Z$ and $\mathcal{W}_{j, l}=\overline{\operatorname{span}_{k} \psi_{j, k, l}} ; \forall k \in Z$ [12]. It follows from (1) that the subspaces $\mathcal{V}_{j}$ have a nested property given by $\mathcal{V}_{j}=\mathcal{V}_{j+1} \oplus\left[\oplus_{l=1}^{M-1} \mathcal{W}_{j+1, l}\right]$. Thus, a function $f(x) \in l^{2}$ can be expressed in terms of the sum of projections onto subspaces $\mathcal{V}_{j}$ and $\mathcal{W}_{j, l}$ as

$$
f(x)=\sum_{k} c(k) \phi_{j, k}(x)+\sum_{l=1}^{M-1} \sum_{k} d_{l}(k) \psi_{j, k, l}(x) .
$$

We can extend our discussion in defining wavelet packets as a generalization of orthonormal and compactly supported wavelets [13]. From the subband filtering point of view, the difference between the wavelet packet transform (DWPT) and the standard wavelet transform (DWT) is that the former recursively decomposes the high-frequency components as well, unlike the other, thus resulting in a tree-structured multiband extension of the wavelet transform.

\section{WAVELET FEATURE EXTRACTION}

The methodology for feature extraction involves $M$-band wavelet packet filtering of an input image followed by adaptive basis selection. Subsequently, features are computed from this set of selected basis by using a nonlinear operator and smoothing filter.

\section{A. M-Band Wavelet Packet Filters and Adaptive Basis Selection}

The objective of filtering is to transform the edges in a texture image into detectable discontinuities. The filter bank in essence is a set of bandpass filters that select frequency and orientation. In the filtering stage, we make use of orthogonal and linear phase $M$-band $(M=4)$ wavelets following [13].

The motivation for the use of larger $M(M>2)$ is to have a more flexible tiling of the time-frequency (scale-space) plane than that resulting from the two-band wavelet. It also enables to have some regions of uniform bandwidths rather than the logarithmic spacing of the frequency responses. Although the $M$-band wavelet decomposition results in a combination of linear and logarithmic frequency (scale) resolution, we conjecture that a further recursive decomposition of the high-frequency regions would characterize textures better.

The $M$-band wavelet decomposition can be interpreted as signal decomposition in a set of independent spatially oriented frequency channels. Discrete $M$-band wavelet frame (DMbWF) decomposition is similar to the discrete $M$-band wavelet transform (DMbWT), except that no down-sampling is done over different scales (levels of decomposition). This is done to achieve invariance to translation. It is worth mentioning that other alternatives are also available to tackle this problem of shift (translation) variance by using complex wavelets [16].

The discrete $M$-band wavelet packet transform (DMbWPT) can be looked upon as a generalization of DMbWT. At scale $j=J$, the image is first decomposed into $M \times M$ channels using all the filters $h_{j}$ and $g_{j, l}$ with $l=1,2,3$ and without down-sampling. The process is repeated for each of the subbands for subsequent scales.

For extraction of textural features of a remotely sensed image, it is appropriate to detect the most significant frequency bands contained in the image. This leads naturally to a tree-structured wavelet transform of the image. An $M$-band wavelet packet decomposition gives $M^{2^{k}}$ number of bases, for a decomposition depth of $k$. It is usually redundant to decompose all the subbands in each scale to achieve the full tree of decomposition. Also, it is quite evident that an exhaustive search to determine the optimal basis from this large set is computationally expensive.

In order to find out a suitable basis without going for a expensive full decomposition, we make use of an adaptive decomposition algorithm using a maximum criterion of textural measures 
extracted from each of the subbands [17]. Then the most significant subbands are identified, and it is decided whether further decomposition of the particular channel would generate more information or not. This search is computationally efficient and enables one to zoom into any desired frequency channel for further decomposition [18].

After decomposition of the image into $M \times M$ channels as described above, the energy for each subband is evaluated. Among these subbands, those for which energy values exceed $\epsilon_{1} \%$ of the energy of the parent band are considered and decomposed further. A subband is further decomposed if its energy value is more than some $\epsilon_{2} \%$ of the total energy of all the subbands at the current scale. This step results in a set of four-band wavelet packet bases. These bases corresponding to different resolutions are assumed to capture and characterize effectively different scales of texture of the input image. Empirically we have seen that a value of $\epsilon_{1}=2 \%$ and $\epsilon_{2}=10 \%$ are a good choice for the images we have considered here.

\section{B. Computation of Features}

After selection of the significant bases, a local estimator that constitutes a nonlinear operator followed by a smoothing filter is applied to each subband. This estimates a textural feature of a subband image in a local region around each pixel. A nonlinearity is needed in order to discriminate texture pairs with identical mean brightness and second-order statistics. Since the magnitude of the correlation between the wavelet function and the image is all that is important, the absolute values of the wavelet coefficients are used as a generalized energy definition.

For a subband image $F_{s}(x, y)$ of subband number $s$, where $0 \leq x \leq M-1,0 \leq y \leq N-1$, the local energy $\operatorname{Eng}_{s}(x, y)$ around the $(x, y)$ th pixel can be formally defined as $\operatorname{Eng}_{s}(x, y)=\left|F_{s}(m, n)\right|$. This step is succeeded by a Gaussian lowpass filter $h_{G}(x, y)$ to get a feature image. Formally, the feature image Feat $_{s}(x, y)$ corresponding to subband image $F_{s}(x, y)$ is given by Feat $_{s}(x, y)=\sum_{(a, b) \in G_{x y}} \Gamma\left(F_{s}(a, b) h_{G}(x-a, y-b)\right)$, where $\Gamma(\cdot)$ gives the energy measure and $G_{x y}$ is a $G \times G$ window centered at pixel with coordinates $(x, y)$.

\section{NEUROFUZZY FEATURE EVALUATION}

The wavelet features extracted in Section III are now evaluated in a neurofuzzy framework under unsupervised learning. The present method is a modification of an earlier method [19]. This modification enables one to handle large datasets in a more efficient manner.

\section{A. Fuzzy Feature Evaluation Index and Membership Function}

The feature evaluation index for a set of transformed features is defined as

$$
E=\frac{2}{s(s-1)} \sum_{p} \sum_{q \neq p} \frac{1}{2}\left[\mu_{p q}^{T}\left(1-\mu_{p q}^{O}\right)+\mu_{p q}^{O}\left(1-\mu_{p q}^{T}\right)\right]
$$

Here $\mu_{p q}^{O}$ and $\mu_{p q}^{T}$ are the degree that both the $p$ th and $q$ th patterns belong to the same cluster in the $n$-dimensional original feature space, and in the $n^{\prime}$-dimensional $\left(n^{\prime} \leq n\right)$ transformed feature space, respectively. $\mu$ values determine how similar a pair of patterns are in the respective features spaces. $s$ is the number of samples on which the feature evaluation index is computed.

The feature evaluation index decreases as the membership value representing the degree of belonging of $p$ th and $q$ th patterns to the same cluster in the transformed feature space tends to either 0 (when $\mu^{O}<0.5$ ) or 1 (when $\mu^{O}>0.5$ ). Therefore, our objective is to select those features for which the evaluation index becomes minimum, thereby optimizing the decision on the similarity of a pair of patterns with respect to their belonging to a cluster.

The membership function $\mu_{p q}$ in a feature space, satisfying the characteristics of $E$ in (4), may be defined as [19]

$$
\begin{aligned}
\mu_{p q} & =1-\frac{d_{p q}}{D}, & & \text { if } d_{p q} \leq D \\
& =0, & & \text { otherwise }
\end{aligned}
$$

where the distance $d_{p q}$ between the $p$ th and $q$ th patterns can be written as

$$
\begin{aligned}
d_{p q} & =\left[\sum_{i} w_{i}^{2}\left(x_{p i}-x_{q i}\right)^{2}\right]^{\frac{1}{2}} \\
& =\left[\sum_{i} w_{i}^{2} \chi_{i}^{2}\right]^{\frac{1}{2}} \cdot \chi_{i}=\left(x_{p i}-x_{q i}\right) .
\end{aligned}
$$

The term $w_{i} \in[0,1]$ represents weighting coefficient corresponding to the $i$ th feature, and $x_{p i}$ and $x_{q i}$ are values of the $i$ th feature (in the corresponding feature space) of the $p$ th and $q$ th patterns, respectively. $D$ is a parameter that reflects the minimum separation between a pair of patterns belonging to two different clusters [19] and is expressed as $D=\alpha d_{\max }$, where $d_{\max }$ is the maximum separation between a pair of patterns in the entire feature space, and $0<\alpha \leq 1$ is a user-defined constant. $\alpha$ determines the degree of flattening of the membership function (5). $d_{\max }$ is defined as $d_{\max }=\left[\sum_{i}\left(x_{\max i}-x_{\min i}\right)^{2}\right]^{(1 / 2)}$, where $x_{\max i}$ and $x_{\min i}$ are the maximum and minimum values of the $i$ th feature in the corresponding feature space.

The membership value $\mu_{p q}$ is dependent on $w_{i}$. The values of $w_{i}(<1)$ make the $\mu_{p q}$ function of (5) flattened along the axis of $d_{p q}$. The weight $w_{i}$ in (6) reflects the relative importance of the feature $x_{i}$ in measuring the similarity of a pair of patterns. The higher the value of $w_{i}$, the more is the importance of $x_{i}$ in characterizing a cluster or discriminating various clusters. $w_{i}=$ $1(0)$ indicates most (least) importance of $x_{i}$.

The evaluation index $E$ in (4) is a function of $\mathbf{w}$, if we consider ranking of $n$ features in a set. The problem of feature selection/ranking, thus, reduces to finding a set of $w_{i} \mathrm{~s}$ for which $E$ becomes minimum. The task of minimization, as in [19], is performed using gradient-descent technique in a connectionist framework under unsupervised mode (for details please see [19]).

\section{B. Modified Neurofuzzy Algorithm for Handling Large Data}

The number of patterns generated for an $N \times N$ image is $N^{2}$, i.e., $s=N^{2}$. Each of these patterns corresponds to a 


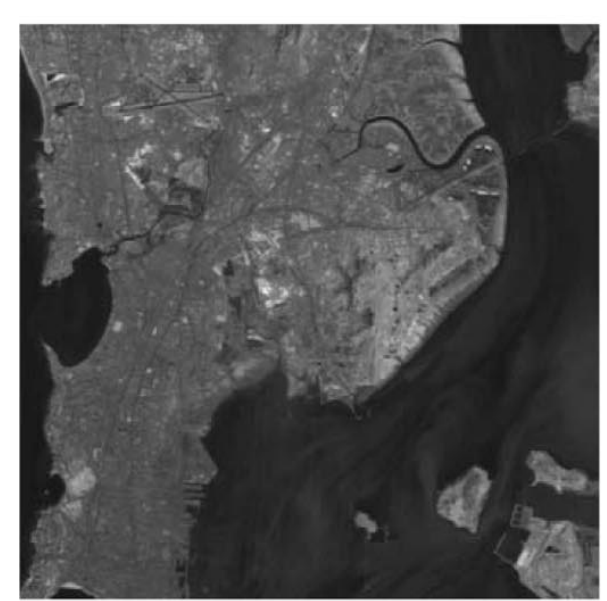

(a)

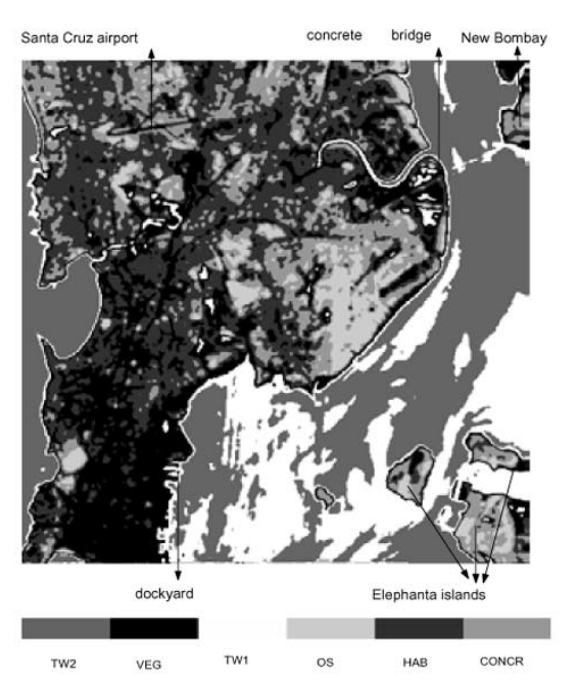

(b)

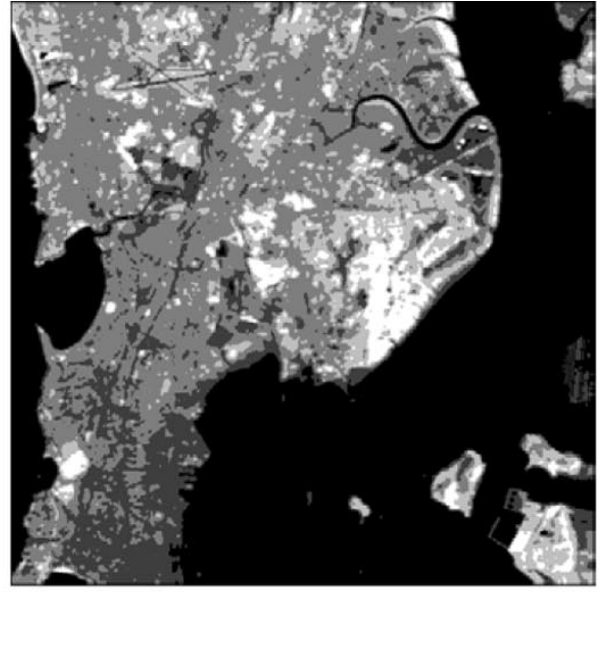

(c)

Fig. 1. (a) Bombay IRS-1A image (band 4) segmented output with $c=6$. (b) With neurofuzzy feature evaluation $(\beta=8.21309$ ). (c) With average and busyness as features $(\beta=7.21309)$.

pixel and has all the multiscale wavelet feature extracted in Section III. Therefore, for selecting an optimal set of features out of them, the number of patterns to be presented to the connectionist system in one epoch, during its training, is $(s(s-$ 1)) $/(2)=\left(N^{2}\left(N^{2}-1\right)\right) /(2)$, which is a very large quantity [19]. This incurs a very high computational time. In order to make the neurofuzzy algorithm computationally more efficient, we, first of all, apply a clustering algorithm (e.g., $k$-means clustering algorithm) on the entire feature space, for grouping the data, and the cluster centers $\boldsymbol{c e n}_{q}$ 's are noted. Then, two sets of samples, namely, $S=\left\{\boldsymbol{x}_{1}, \boldsymbol{x}_{2}, \ldots, \boldsymbol{x}_{p}, \ldots, \boldsymbol{x}_{N^{2}}\right\}$ and $S_{c}=\left\{\boldsymbol{c e n}_{1}, \boldsymbol{c e n}_{2}, \ldots, \boldsymbol{c e n}_{c}\right\}$ are formed. That is, $S$ is the entire training set, and $S_{c}$ is the set of $c$ cluster centers (for $c$ clusters) obtained by the clustering algorithm. Now, the similarity between the patterns and these cluster centers are computed, instead of computing it for every pair of patterns. These cluster centers are considered as representatives (prototypes) of all the points belonging to the respective clusters. Thus, the number of patterns to be presented to the network in one epoch becomes $\left(s\left(s_{c}-1\right)\right) /(2)=\left(N^{2}(c-1)\right) /(2)$, where $s=|S|$ and $s_{c}=\left|S_{c}\right| \ll s$.

$E$ [in (4)] after convergence attains a local minimum and then the weights $\left(W_{j}=w_{j}^{2}\right)$ of the links connecting hidden nodes and the output node computing $\mu^{T}$ values, indicate the order of importance of the features. Note that this unsupervised method performs the task of feature selection without clustering the feature space explicitly and does not need to know the number of clusters present in the feature space. We have used a traditional $k$-means clustering algorithm [20].

\section{Segmentation of IRS AND SPOT Images}

Here we demonstrate the effectiveness of our methodology over several remotely sensed images of size $512 \times 512$.

The IRS-1A (Indian Remote Sensing 1A) satellite image has four bands, and in Fig. 1(a) we show the band-4 image of a part of the city of Bombay. The image consists of six major classes, viz., turbid water 1 (TW1), turbid water 2 (TW2), concrete (CONCR), habitation (HAB), vegetation (VEG), and open spaces (OS). The sea water is decomposed into two classes TW1 and TW2 for better classification, since they have somewhat different reflectance properties due to variation in sea water density [as seen in Fig. 1(a)].

The SPOT image [Fig. 2(a)] shows a portion of Calcutta in one of its three bands. This image has seven major classes, which are turbid water (TW), pond water ( $\mathrm{PW})$, concrete (CONCR), vegetation (VEG), habitation (HAB), open space (OS), and roads/bridges (B/R).

In order to validate the importance of neurofuzzy feature evaluation, we show how the feature dimensionality can be reduced greatly after feature evaluation. The test images have several fine (line) structures (roads and bridges). In order to detect these structures, the window size has been kept small $(3 \times 3)$. A quantitative index $\beta$ [21] is used to evaluate the segmentation results. $\beta$ is defined as the ratio of the total variation and within-class variation. The higher the value of $\beta$, the better is the segmentation.

The $\beta$ value of the IRS-1A image of Bombay, considering the number of classes to be $c=6$, is found to be 8.01462 . The number of extracted features from all four bands is found to be 12 as obtained by Section III. The feature dimensionality is reduced to one after neurofuzzy feature evaluation, and the $\beta$ index (8.213 09) shows better segmentation quality. In the segmented output after the feature evaluation step [Fig. 1(b)], it can be seen that the dockyard, the concrete structure connecting Bombay and New Bombay, and the Santa Cruz airport are very well detected. The feature evaluation step increases the compactness of the various classes. For a comparative study, the segmentation result in Fig. 1(c) is obtained by using $k$-means clustering algorithm with average and busyness as features. Here also we can find an improvement in segmentation quality using our algorithm.

In the case of SPOT image of Calcutta [Fig. 2(a)], we have initially considered all ten features extracted by the wavelet-based method. The segmentation result with $c=7$ gives a $\beta$ value of 


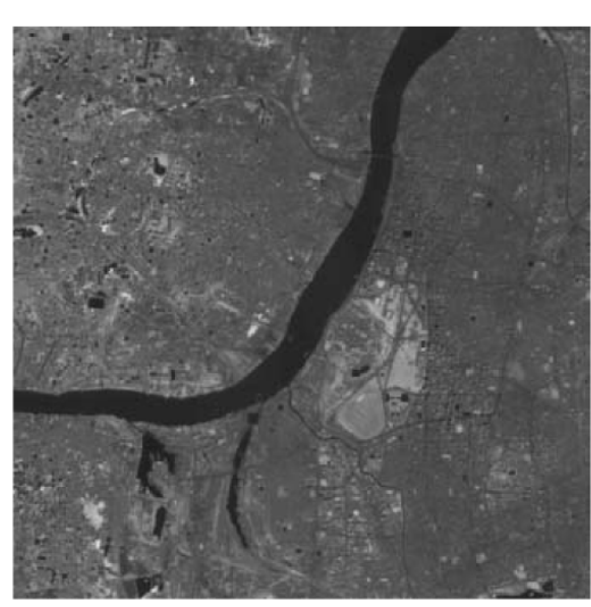

(a)

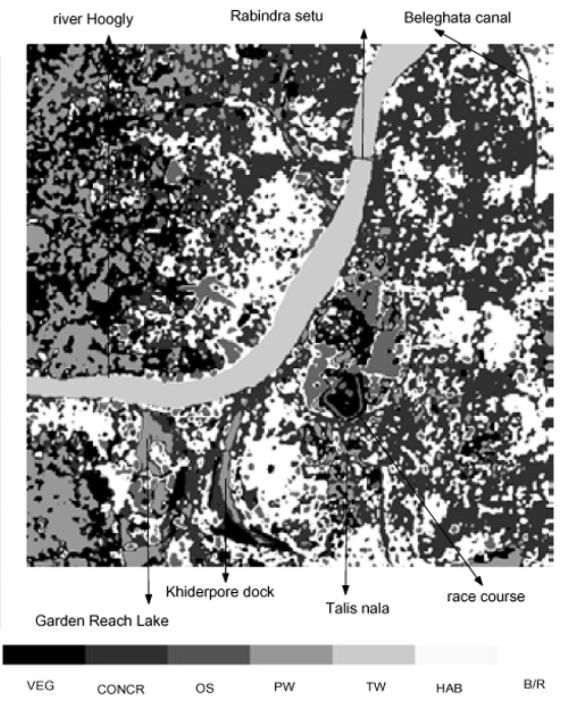

(b)

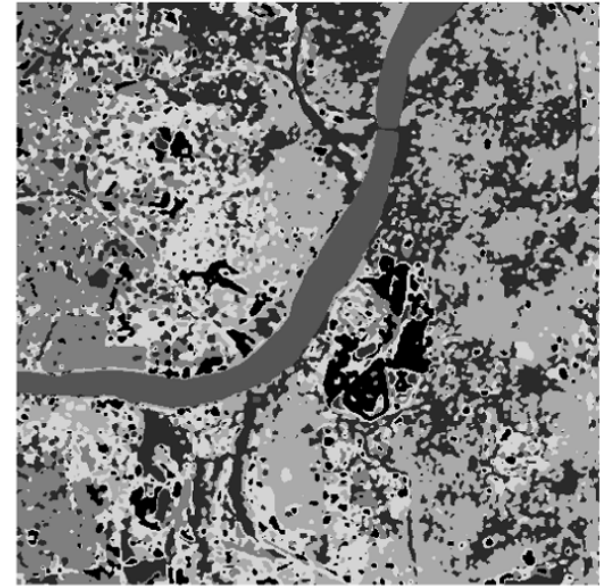

(c)

Fig. 2. (a) Calcutta SPOT image (band 3) segmented output with $c=7$. (b) With neurofuzzy feature evaluation ( $\beta=3.45631$ ). (c) Using average and busyness as features $(\beta=2.45631)$

3.278 32. After the neurofuzzy feature evaluation, the number of features reduces to three. The segmentation result is given in Fig. 2(b), and the $\beta$ value (3.45361) indicates better segmentation quality. It is to be noted that not only the race course but also a triangular outline (which is an open space) corresponding to the track of the race course are also detected. Here the Rabindra Setu has also been detected along with the Talis nala and Beleghata canal. Moreover, the Khiderpore dock and Garden Reach Lake are quite discernible. In this cases, all three bands are important and furnish some information. The features obtained after the feature evaluation step correspond to each of the three bands. The segmentation result along with the $\beta$ value obtained by using $k$-means clustering algorithm with average and busyness as features (as mentioned above) is given in Fig. 2(c) for a comparative study validating that the features extracted by our method characterize the remotely sensed images in a better way.

In order to prove the efficacy of our algorithm, we have tested it on a synthetic texture images, and results are shown in [17]. Interestingly, the proposed methodology has been able found to be superior to various existing related algorithms [17].

\section{CONCLUSION}

In this paper, we have presented a scheme to show how wavelet theory and neurofuzzy hybridization together can be applied in the domain of remotely sensed imagery for segmentation purpose. The use of wavelet theory via $M$-band wavelet decomposition of remotely sensed images provides an efficient representation of these images in terms of frequencies in different directions and orientations at different resolutions. This representation is obtained by segmenting some wavelet features from multispectral remotely sensed images. This facilitates an improved segmentation quality of the different class regions. The feature extraction method splits the lower as well as the higher frequency bands and results in a tree structure. This enables the system to extract their characteristic features in the lower as well as the higher frequency bands of remotely sensed images. The neurofuzzy feature evaluation method helps in searching for important features efficiently from a remotely sensed image where the various classes are overlapping in nature. Both the feature extraction and neurofuzzy feature evaluation schemes are unsupervised and do not require any a priori knowledge about the number of classes, and spatial relationship of different regions in these images. It also shoudl be noted that the neurofuzzy feature evaluation tchnique presented here is a modified version of an existing method described in [19], which is able to deal with a large dataset more efficiently. The algorithm has also been tested on synthetic data comprising natural textures. The results validate [17] that the proposed methodology is indeed superior to some of the related ones existing in the literature. Also, the results presented using average and busyness as features and using the $k$-means clustering algorithm for a comparative study show that our methodology is indeed effective in characterizing remotely sensed images in a better way.

It is found that for the IRS-1A image, ultimately one feature is selected that corresponds to band 4 of this image. However, for the SPOT image, three features are selected. In all cases, almost all the desired classes are obtained in the segmented output satisfactorily. Moreover, the compactness of the various classes increases after the neurofuzzy feature evaluation step.

\section{REFERENCES}

[1] M. Tuceryan and A. K. Jain, "Texture analysis," in Handbook of Pattern Recognition and Computer Vision, C. H. Chen, L. F. Pau, and P. S. P. Wang, Eds, Singapore: World Scientific, 1993, ch. 2, pp. 235-276.

[2] R. M. Haralick, K. Shanmugam, and I. Dinstein, "Texture feature for image classification," IEEE Trans. Syst., Man Cybern., vol. SMC-8, no. 6, pp. 610-621, 1973.

[3] E. Rignot and R. Kowk, "Extraction of textural features in sar images: Statistical model and sensitivity," in Proc. IGARSS, May 1990.

[4] L. J. Du, "Texture segmentation of sar images using localized spatial filtering," in Proc. IGARSS, 1990, pp. 1983-1986. 
[5] A. Mecocci, P. Gamba, A. Marazzi, and M. Barni, "Texture segmentation in remote sensing images by means of packet wavelets and fuzzy clustering," in Proc. SPIE Eur. Symp. Satellite and Remote Sensing II, vol. 2584, Sept. 1995, pp. 142-157.

[6] R. W. Lindsay, D. B. Percival, and D. A. Rothrock, "The discrete wavelet transform and the scale analysis of the surface properties of sea ice," IEEE Trans. Geosci. Remote Sensing, vol. 34, pp. 771-787, May 1996.

[7] S. Fukuda and H. Hirosawa, "A wavelet-based texture feature set applied to classification of multifrequency polarimetric sar images," IEEE Trans. Geosci. Remote Sensing, vol. 37, pp. 2282-2286, Sept. 1999.

[8] M. Simard, S. S. Saatchi, and G. De Grandi, "The use of decision tree and multiscale texture for classification of JERS-1 sar data over tropical forest," IEEE Trans. Geosci. Remote Sensing, vol. 38, pp. 2310-2321, Sept. 2000.

[9] A. Niedermeier, E. Romaneesen, and S. Lehner, "Detection of coastline SAR images using wavelet methods," IEEE Trans. Geosci. Remote Sensing, vol. 38, pp. 2270-2281, Sept. 2000.

[10] P. Thitimajshima, "Multiresolution fuzzy clustering for sar image segmentation," in Proc. IGARSS, vol. 5, 1999, pp. 2507-2509.

[11] M. Unser, "Texture classification and segmentation using wavelet frames," IEEE Trans. Image Processing, vol. 4, pp. 1549-1560, Nov. 1995.

[12] P. Steffen, P. N. Heller, R. A. Gopinath, and C. S. Burrus, "Theory of regular $M$-band wavelet bases," IEEE Trans. Signal Processing, vol. 41, pp. 3497-3510, Dec. 1993.

[13] O. Alkin and H. Caglar, "Design of efficient $M$-band coders with linear phase and perfect reconstruction properties," IEEE Trans. Signal Processing, vol. 43, pp. 1579-1590, July 1995.

[14] S. K. Pal, A. Ghosh, and M. K. Kundu, Eds., Soft Computing for Image Processing. Heidelberg, Germany: Physica Verlag, 2000.

[15] H. Zou and A. H. Tewfik, "Discrete orthogonal, $M$-band wavelet decomposition," in Proc. Int. Conf. Acoustics, Speech, and Signal Processing, vol. 4, 1992, pp. IV-605-IV-608.

[16] N. G. Kingsbury, "Complex wavelets and shift invariance," in Proc. Inst. Elect. Eng. Colloq. Time-Scale and Time-Frequency Analysis and Applications, 2000.

[17] M. Acharyya and M. K. Kundu, "Adaptive basis selection for multitexture segmentation by $m$-band wavelet packet frame," in Proc. Int. Conf. Image Processing, Sept. 2001, pp. 622-625.

[18] M. Acharyya and M. K. Kundu, "Wavelet-based texture segmentation of remotely sensed images," in Proc. Int. Conf. Image Analysis and Processing, Sept. 2001, pp. 69-74.

[19] S. K. Pal, R. K. De, and J. Basak, "Unsupervised feature evaluation A neuro-fuzzy approach," IEEE Trans. Neural Networks, vol. 11, pp. 366-376, Mar. 2000.

[20] J. T. Tou and R. C. Gonzales, Pattern Recognition Principles. Reading, MA: Adision-Wesley, 1974

[21] S. K. Pal, A. Ghosh, and B. Uma Shankar, "Segmentation with remotely sensed images with fuzzy thresholding, and quantitative evaluation," Int J. Remote Sens., vol. 21, no. 11, pp. 2269-2300, 2000.

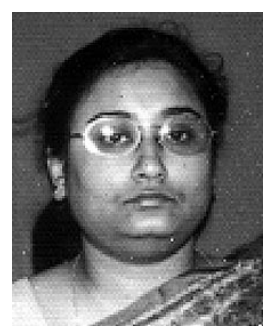

Mausumi Acharyya received the B.Sc. degree in physics, the B.Tech. and M.Tech. degrees in radiophysics and electronics, all from the University of Calcutta, Calcutta, India, and the Ph.D. degree in computer science from the Indian Statistical Institute, Kolkata, India, in 2003.

She is currently a Scientist-D at the Electronics and Radar Development Establishment, Defence Research Development Organization, Bangalore, India. From 1995 to 2002, she was with the Machine Intelligence Unit, Indian Statistical Institute. Her current research interests are in the areas of image processing and analysis, pattern recognition, wavelets and soft computing, medical imaging, bioinformatics, and imaging radar.

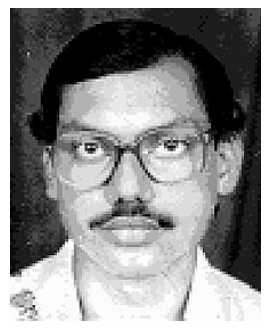

Rajat K. De (M'03) received the B.Tech from the Univeristy of Calcutta, Calcutta, India, in 1991, the M.E. degree from the Jadavpur University, Jadavpur, India, in 1993, and the Ph.D. degree from the Indian Statistical Institute, Calcutta, India, in 2000, all in computer science.

$\mathrm{He}$ is currently an Assistant Professor in the Machine Intelligence Unit, Indian Statistical Institute. His research interests include pattern recognition, image processing, fuzzy sets, neural networks, soft case-based reasoning. computing, wavelet theory, bioinformatics, and

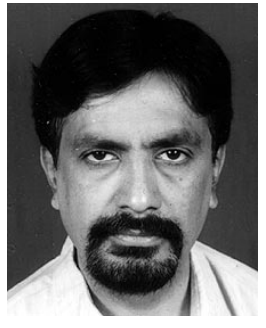

Malay K. Kundu (M'90-SM'99) received the B. Tech., M. Tech., and Ph.D. (Tech.) degrees from the University of Calcutta, Calcutta, India, all in radio physics and electronics.

In 1982, he joined the Indian Statistical Institute, Calcutta, as a Faculty Member. He had been the Head of the Machine Intelligence Unit, Indian Statistical Institute from September 1993 to November 1995 and is currently a Full Professor there. His current research interests include image processing and analysis, image compression, machine vision, genetic algorithms, fractals, wavelets, VLSI design for digital imaging, and soft computing. He has contributed about 70 research papers in well-known and prestigious archival journals, international refereed conferences, and as chapters in monographs and edited volumes. He is coauthor of the book titled Soft Computing for Image Processing (Heidelberg, Germany: Physica-Verlag).

Dr. Kundu is a Fellow of the National Academy of Sciences and a Fellow of the Institute of Electronics and Telecommunication Engineers. He received the prestigious VASVIK Award for industrial research in Electronic Sciences and Technology in 1999 and the Sir J. C. Bose Memorial Award in 1986. 